\title{
Tuning sedimentation through surface charge and particle shape
}

\author{
Ali Seiphoori ${ }^{1,2}$, Andrew Gunn ${ }^{1}$, Sébastien Kosgodagan Acharige ${ }^{3}$, Paulo E. \\ Arratia $^{3}$ and Douglas J. Jerolmack ${ }^{1,3}$ \\ ${ }^{1}$ Department of Earth and Environmental Science, University of Pennsylvania, Philadelphia, \\ Pennsylvania, PA 19104, USA. \\ ${ }^{2}$ Department of Earth, Atmospheric, and Planetary Sciences, Massachusetts Institute of Technology, MA \\ 02139, USA \\ ${ }^{3}$ Department of Mechanical Engineering and Applied Mechanics, University of Pennsylvania, Philadelphia, \\ Pennsylvania, PA, 19104, USA.
}

Key Points:

- Aggregation of clay particles enhances sedimentation and forms mud in nature.

- Changing surface charge results in a phase transition from hindered settling to a collapsing gel behavior.

- Formation and collapse of clay gels may be important for coastal erosion.

Corresponding author: Ali Seiphoori, aliseiph@mit.edu

This is the author manuscript accepted for publication and has undergone full peer review but has not been through the copyediting, typesetting, pagination and proofreading process, which may lead to differences between this version and the Version of Record. Please cite this article as doi: 10.1029/2020GL091251.

This article is protected by copyright. All rights reserved. 


\begin{abstract}
Mud forms the foundation of many coastal and tidal environments. Clay suspensions carried downstream from rivers encounter saline waters, which encourages aggregation and sedimentation by reducing electrostatic repulsion among particles. We perform experiments to examine the effects of surface charge on both the rate and style of sedimentation, using kaolinite particles as a model mud suspension and silica spheres with equivalent hydrodynamic radius as a control. Classic hindered settling theory reasonably describes sedimentation rate for repulsive clay particles and silica spheres, which form a highly concentrated jamming front. The hindered settling description breaks down for attractive clay particles, which aggregate to form clay gels that consolidate like a soft solid. Water flow form fracture-like channels in the bulk of the gel, which disappear as gel enters a creep regime. Results may help toward understanding the effect of surface charge and particle shape on the sedimentation and erodibility of natural mud.
\end{abstract}

\title{
Plain Language Summary
}

When suspended sediment is transported from land to the ocean by river, the water surrounding the sediment particles changes from fresh to salty. This change creates increased interparticle attraction, leading sediment to aggregate and deposit. In contrast to ocean salinity, artificial fertilizers may contain different salts that have the opposite effect on interparticle forces, creating repulsion that suppresses aggregation. These chemical effects, and the way particles sink, are modulated by the shape of the sediment too. Here we perform experiments to examine these effects on sedimentation, using kaolinite particles as a model mud suspension and glass beads as a control. We see that how the particles sink is sensitive to chemistry: when they are repulsive a classic 'hindered settling' theory predicts their deposition well, and when attractive the particles link up in a network that behaves like a single structure that collapses under its own weight. The flow of water out of the structure - which we call a gel - as it collapses becomes localized into fracture-like channels that disappear as the deformation slows down and the gel gets denser. Our observations improve understanding of mud sedimentation, which is essential to predict how estuaries and coastal environments change.

\section{Introduction}

Silt and clay sized particles are well suspended in rivers. On approach to the ocean, however, where river currents slow down and begin to mix with saline waters, these particles deposit to form mud. The screening of repulsive surface charges by dissolved salt ions facilitates clay aggregation (or flocculation), which greatly enhances sedimentation rates by increasing effective particle mass (Coussot \& Piau, 1994; Winterwerp, 2002; Whitehouse et al., 2013; Sutherland et al., 2015). This effect helps to build marsh platforms and river deltas, tidal channels and estuaries, and even the continental slope (Figure 1A). Clay aggregation also influences the deposition rate, and likely the runout, of muddy turbidity currents that deliver sediment from the continental shelf to deep marine environments (Packman \& Jerolmack, 2004). As sticky aggregates sink to the bottom of the water column and particle volume fraction $\phi$ increases, a curious transition occurs: clusters join to form one large interconnected network, and the suspension develops an effective yield stress (Allain et al., 1995; Manley et al., 2005; Dankers \& Winterwerp, 2007; Ali \& Bandyopadhyay, 2016). Under continued sedimentation in a quiescent fluid, this "house of cards" structure eventually collapses to produce a consolidated mud deposit (Toorman \& Berlamont, 1991; Dankers \& Winterwerp, 2007; Bartlett et al., 2012; Teece et al., 2014). In the presence of shear, however, this "fluid mud" suspension may persist indefinitely in a quasi-stable state until some perturbation causes it to suddenly yield and flow (Traykovski et al., 2000; Heymann et al., 2002; McAnally et al., 2007; Mueller et al., 2010; Talling et al., 2012; Nie et al., 2020; Jerolmack \& Daniels, 2019). 


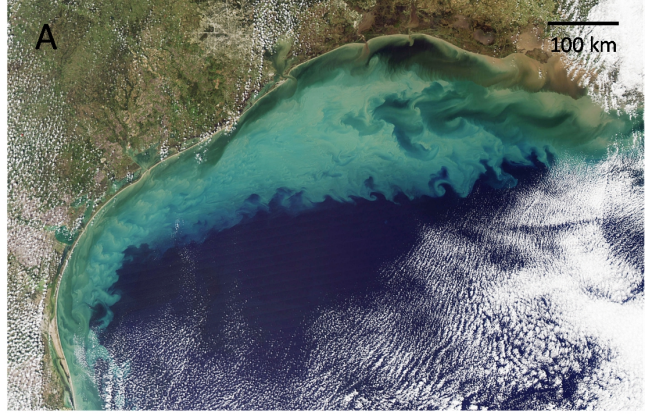

B

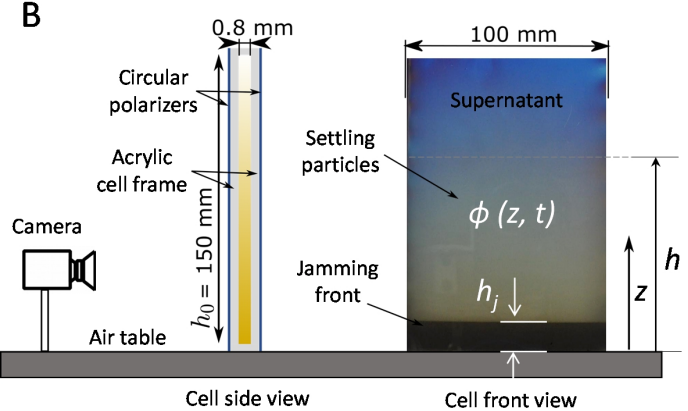

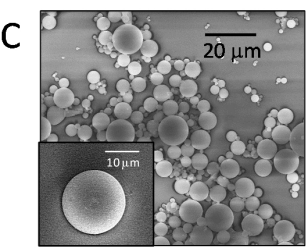

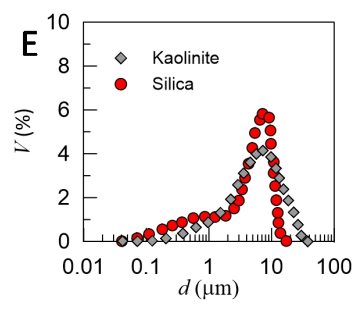

D

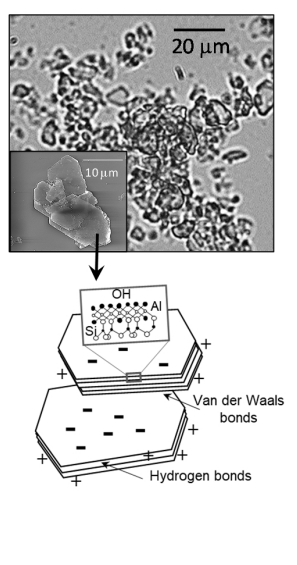

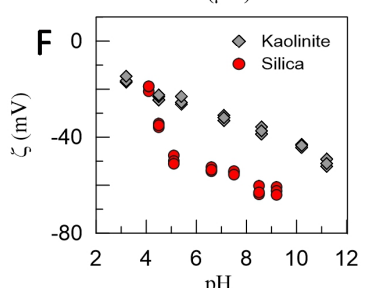

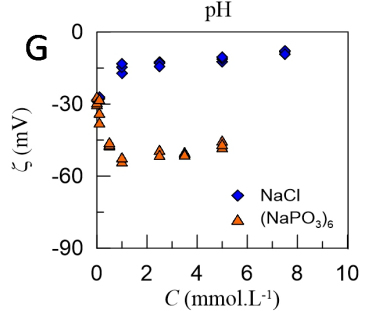

Figure 1. (A) Satellite image of the Mississippi River entering the Gulf of Mexico, showing muddy coastal water (image courtesy of NASA Earth Observatory). (B) Experimental setup used to study sedimentation. The image is analogous to the experiment of repulsive kaolinite particles with volume fraction $\phi=1.6 \%$. (C)-(D) Morphology of the polydisperse silica spheres and kaolinite clay particles, respectively. (E) Particle size distribution of the sieved kaolinite particles and the polydisperse silica microspheres model system. (F) Zeta potential $(\zeta)$ of kaolinite particles and silica microspheres at various $\mathrm{pH}$ conditions. $(\mathbf{G})$ Zeta potential $(\zeta)$ of kaolinite particles as a function of salt concentration, for sodium hexametaphosphate $\left(\mathrm{NaPO}_{3}\right)_{6}$ and sodium chloride $\mathrm{NaCl}$.

It is hypothesized that fluid muds are a kind of particulate gel (Coussot, 2017; Dankers \& Winterwerp, 2007; Talling et al., 2012; Ali \& Bandyopadhyay, 2016), in which percolated clusters of aggregates form a soft, metastable solid at much lower values of $\phi$ than is observed for repulsive particles. The stability of particulate gels depends on the strength of the interparticle bonds. In weak gels, the attractive potential at interparticle contact is estimated to be several times the value of $k_{B} T$ (where $k_{B}$ is Boltzmann's constant and $T$ is temperature); this results in a delayed collapse of the structure (Manley et al., 2005; Gopalakrishnan et al., 2006; Bartlett et al., 2012; Teece et al., 2014; Ali \& Bandyopadhyay, 2016). The delay time depends on different factors including the strength of the interparticle bonds, the particle geometry, and the volume fraction $\phi$ (Buscall et al., 2009). After the onset of collapse, channels form in the bulk of the gel due to an upward flow of the solvent as it drains (Allain et al., 1995; Derec et al., 2003; Buscall et al., 2009; Marr et al., 2001). Transient collapse of colloidal gels was first reported by Poon et. al. (1993), and has been studied in: depletion-induced gels (Starrs et al., 2002a; Bartlett et al., 2012; Teece et al., 2014); gels formed by van der Waals attraction (Allain et al., 1995; Manley et al., 2005; Buscall et al., 2009; Ali \& Bandyopadhyay, 2016); and colloidal gels flocculated at their secondary minima (Bergström, 1992). For the most part, recent advances in the physics of colloidal gel formation and collapse have not yet made contact with geological/geotechnical investigations of mud, which use separate frameworks to model the consolidation behavior of saturated clays (Biot, 1941). Distinct regimes of clay gel sta- 
bility and rheology may be found within the range of salt concentrations encountered in nature; salt influences (i) the interfacial potentials, and (ii) strength of the interparticle bonds between clay particles. Despite recent progress, the microscopic origins and mechanisms of transient collapse behavior in clay gels remain poorly understood. One important question is how surface charge and particle shape affect the sedimentation of clay particles.

In this study we perform experiments to isolate the control of surface charge on both the rate and style of sedimentation of a model mud. We tune the surface potential of kaolinite clay suspensions using inorganic salts, driving them from a classic hindered settling regime to a particulate gel regime. We also examine particle shape effects, by comparing suspensions of clay and silica spheres having similar particle size distribution, surface charge and initial concentration. We find that repulsive kaolinite and silica spheres both form a jammed sedimentation front, but the former settles two orders of magnitude slower than the latter. In contrast, aggregating kaolinite quickly forms a low- $\phi$ gel rather than a jamming front, characterized by a delayed collapse and formation of fracturelike flow channels in the bulk of the gel indicating the dissipation of excess pore pressure. Flow channels disappear with further densification of the gel as it enters a "creeping" regime. We present a constitutive framework to describe the transitional behavior of the gel and show how it relates to the classic consolidation theory. Results illustrate the extreme sensitivity of fine-particle sedimentation to grain properties and solution chemistry, which limits the generality of (site-specific) empirical equations for predicting mud deposition.

\section{Materials and Methods}

Experiments are performed using two different particle types: kaolinite clay particles (particle density $\rho_{k}=2.61 \mathrm{~g} . \mathrm{cm}^{-3}$ ), and polydisperse silica microspheres (particle density $\rho_{s}=2.50 \mathrm{~g} . \mathrm{cm}^{-3}$ ) with comparable size distributions and a modal size of approximately $7 \mu \mathrm{m}$ (Figure 1-E). The zeta potential of kaolinite particles and silica spheres in water at $p H=7.0 \mp 0.5$ are approximately $-30 \mathrm{mV}$ and $-50 \mathrm{mV}$, respectively (Figure 1-F, Supplementary Text S1); sedimentation experiments are performed under $p H \approx$ 7 conditions. The data indicate that silica spheres are more repulsive than kaolinite particles, which tend to aggregate in water. While particle size distributions, surface charge and particle densities are comparable, the particle morphology is not; SEM images show that silica particles are spherical, while kaolinite particles feature a plate-shaped geometry (Figure 1-C and D). Kaolinite particles are tactoids, stacks of single platelets joined together by hydrogen bonds; they typically have an average thickness $\sim 1 \mu \mathrm{m}$ (Johnson \& Kessler, 1969), or about 1/10 of the measured nominal diameter here. Fluid suspensions are prepared by dispersing kaolinite and silica spheres in deionized water at different initial volume fractions, $\phi_{0}$. Adding ions in the suspending liquid results in alteration of the particles' surface charges. Here we manipulate the zeta potential by adding two different salts: sodium chloride, $\mathrm{NaCl}$, and sodium hexametaphosphate, $\left(\mathrm{NaPO}_{3}\right)_{6}$, a known clay de-flocculant, to DI water (Fig. 1-G, Supplementary Text S1). The zeta potential for both suspensions is negative, so that a decrease in value corresponds to increased repulsion. We prepare a range of kaolinite suspensions using varying concentrations of $\left(\mathrm{NaPO}_{3}\right)_{6}, \mathrm{NaCl}$, and initial volume fraction, $\phi_{0}=0.8 \%$ to $4.8 \%$. For experiments with silica spheres we explore $\phi_{0}=4.0$ and $8.0 \%$, but no salts are added. Sedimentation experiments are performed in a transparent cell described in Figure 1-B (Supplementary Text S2). The cell was then placed on the air table for sedimentation to begin. Images were analyzed to determine the volumetric concentration of the suspension throughout the cell, $\phi(z, t)$, the evolution of the sedimenting interface, $h(t)$, and the accumulation front that developed at the bottom of the cell $h_{j}(t)$. We focus in this paper on results from three different suspensions that illustrate the largest range of behaviors: 
1. Silica-R: A silica-sphere suspension in deionized water that is relatively repulsive.

2. Kaolin-R: Kaolinite particles suspended with $10^{-3}$ mol.L $L^{-1}$ sodium hexametaphosphate salt, that is relatively repulsive.

3. Kaolin-A: Kaolinite particles suspended in deionized water and also with $10^{-3} \mathrm{~mol} . \mathrm{L}^{-1}$ sodium chloride solution, that are relatively attractive.

\section{Results and Discussion}

\subsection{Repulsive suspensions}

Figure 2 shows sedimentation of repulsive suspensions: silica- $\mathrm{R}$ with initial volume fraction $\phi_{0}=8.0 \%$ and kaolin-R with $\phi_{0}=1.6 \%$. To quantify the sedimentation process, we plot the interface height $z$ as a function of the normalized volume fraction $\frac{\phi(z)}{\phi_{\max }}$ for several time intervals over the duration of each experiment, where $\phi_{\max }$ is the saturated value of the jamming front; each experiment will yield its own $\phi_{\max }$. We note that these suspensions, which have negligible aggregation, exhibit qualitatively similar behavior that is consistent with classic sedimentation: the falling particles form a sharp density front, resulting from repulsive hydrodynamic interactions as concentration increases, that hinders settling compared to free-fall conditions (Kynch, 1952).

As time progresses, sedimentation produces a diffuse front that travels downward. A clear supernatant forms on the top of the column, followed by a transitional phase of increasing concentration composed of particles with repulsive interactions. Lastly, a jamming front $h_{j}$ emerges at the base of the profile, where volume fraction reaches the saturated value $\phi=\phi_{\max }$ due to deposition; this front migrates upward until the supply of sedimenting particles is exhausted. Similar behavior is seen for all suspensions in which $\zeta<-30 m V$ (Supplementary Table 1), consistent with the typically reported boundary between stable (non-aggregating) and unstable suspensions (Edzwald \& OMelia, 1975). The sigmoidal shape and translating nature of the concentration profiles are similar to previous measurements of thermal and athermal hard-sphere suspensions (Martin et al., 1994), where the width of the sedimentation front is determined by particle dispersivity; this dispersivity results from long-range hydrodynamic interactions between multiple particles, and from thermal motions if colloids are small enough (Brzinski \& Durian, 2018). Despite similar sedimentation styles, rates of deposition are quite different between Silica-R (hours) and Kaolin-R (days) (Fig. 2-B). In the next section we fit a model to the diffusive fronts to understand this difference.

\subsubsection{Modeling sedimentation velocity}

For suspensions of repulsive particles, the sedimentation velocity of each particle depends on the position and velocity of the surrounding particles. This leads to a dependence of the mean sedimentation velocity $U$ on the local particle concentration, i.e., $U=U(\phi)$ with $\phi=\phi(z, t)$ governed by an advection-diffusion equation:

$$
\frac{\partial(\phi U(\phi))}{\partial z}+\frac{\partial \phi}{\partial t}=\frac{\partial}{\partial z}\left(D \frac{\partial \phi}{\partial z}\right)
$$

If one assumes for analytical simplicity that the sedimentation velocity, $U=U(\phi)$, depends linearly on concentration, and that the dispersion coefficient $D$ does not depend on cell height, then the above-mentioned equation becomes a Burgers' equation (Martin et al., 1994). The solution of this equation describes the evolution of the concentration profile at a given time $t$ and height $z$ as follows:

$$
\phi(z, t)=\frac{\phi_{0}}{2}\left[1-\operatorname{erf}\left(\frac{z-t V_{s}}{\sqrt{4 D t}}\right)\right]
$$


A

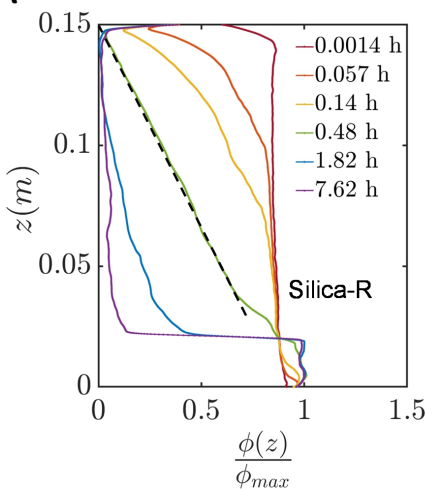

B

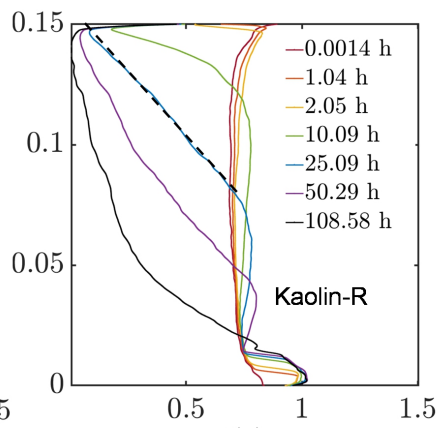

$\frac{\phi(z)}{\phi_{\max }}$

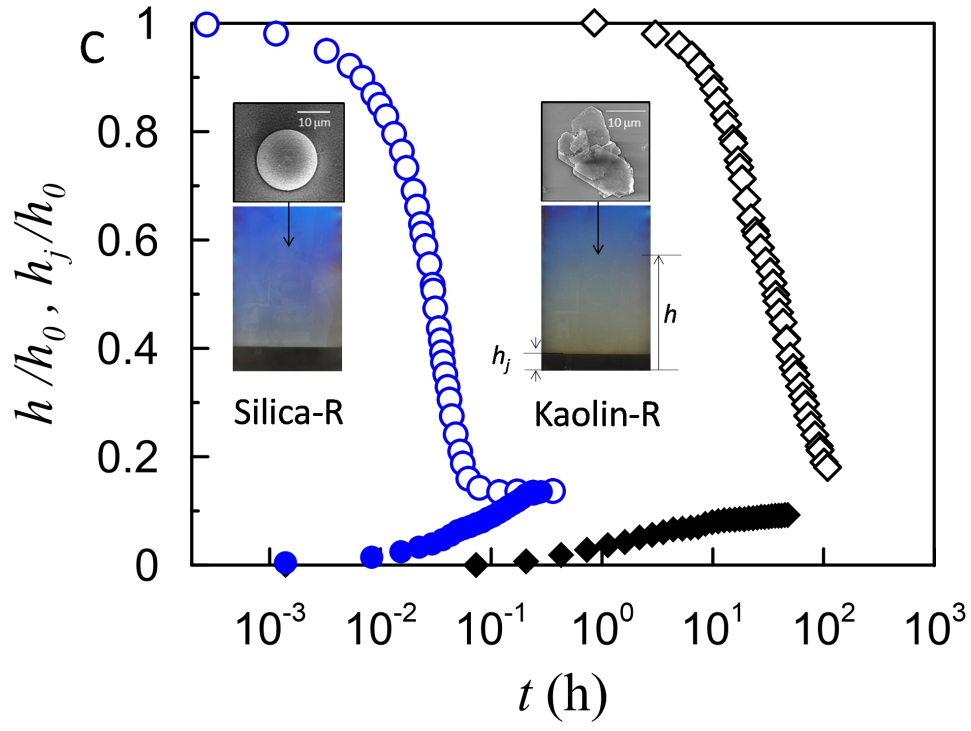

Figure 2. (A), (B) Sedimentation profiles $\frac{\phi(z)}{\phi_{\max }}$, shown at intervals of time marked in legend along the sedimentation height $z$ for Silica-R $\left(\phi_{0}=8 \%\right)$ and Kaolin-R $\left(\phi_{0}=1.6 \%\right)$ suspensions, respectively. The dashed lines in profiles $\mathrm{A}$ and $\mathrm{B}$ are curve fits obtained with equation 2 , with $D$ and $V_{s}$ as fitting parameters. We find $D=4 \times 10^{-5} \mathrm{~m}^{2} \cdot \mathrm{s}^{-1}$ and $V_{s}=6 \times 10^{-5} \mathrm{~m} . \mathrm{s}^{-1}$ for the silica spheres suspension and $D=1.9 \times 10^{-2} \mathrm{~m}^{2} . \mathrm{s}^{-1}$ and $V_{s}=5.5 \times 10^{-7} \mathrm{~m} . \mathrm{s}^{-1}$ for the repulsive kaolinite suspension. (C) Normalized sedimentation front height $\left(\mathrm{h} / \mathrm{H}_{0}\right)$ and jamming front height $\left(\mathrm{h}_{j} / \mathrm{H}_{0}\right)$ against time for both repulsive suspensions

where erf is the error function and $V_{s}=U\left(\phi_{0}\right)$ is the velocity of the sedimentation front. The solution to this equation, with a constant dispersion coefficient $D$, adequately describes the sedimentation profile for the strongly repulsive suspensions (i.e., Silica-R and Kaolin-R) in the middle period of the experiment - i.e., far from the initially uniform condition and the jamming front formation (Fig. 2-A and B). For Silica-R, we find $V_{s} \approx$ $6 \times 10^{-5} \mathrm{~m} \mathrm{~s}^{-1}$ by fitting this model, which is close to the terminal velocity of a solid sphere of diameter $10 \mu m$ determined from the Stokes settling equation, $v_{s}=2 / 9 \times$ $\Delta \rho g r^{2} / \mu$ where $r$ is the particle radius, $\mu$ is the fluid viscosity, $g$ is the gravity acceleration and $\Delta \rho$ is the density difference between silica and water (i.e., $\Delta \rho=1.50 \mathrm{g.cm}-3$ ). The fitted dispersion coefficient $D \approx 4 \times 10^{-5} \mathrm{~m}^{2} \mathrm{~s}^{-1}$ is orders of magnitude larger than the thermal diffusivity for a sphere of diameter $10 \mu \mathrm{m}$ determined from the Stokes-Einstein 
equation $D_{T}=k_{B} T /(6 \pi \mu r)$. This result is expected, at least qualitatively, since hydrodynamic interactions between particles are known to lead to hindering settling (e.g., Brzinski \& Durian, 2018; Guazzelli et al., 2011).

For Kaolin-R suspension, the model fit produces $V_{s}=5.5 \times 10^{-7} \mathrm{~m} . \mathrm{s}^{-1}, 25.09$ hours after sedimentation begins. The fitted value $D=1.9 \times 10^{-2} \mathrm{~m}^{2} \cdot \mathrm{s}^{-1}$ shows that dispersion of repulsive kaolinite particles is three orders of magnitude larger than that of silica spheres. Part of this difference may be accounted for by (Brownian or thermal) diffusivity, which is larger for kaolinite plates due to their smaller mass. Thermal effects cannot, however, explain all of this discrepancy; it is likely that hydrodynamic differences arising from shape-dependent lubrication effects are playing a role (e.g., Le Roux, 2004; Chong et al., 1979).

\subsection{Attractive suspensions}

The sedimentation pattern for Kaolin-A suspension - the least repulsive clay suspension - is distinct from the others, presumably due to aggregation (Fig. 3-A). Although both kaolinite suspensions have the same initial volume fraction $\left(\phi_{0}=1.6 \%\right)$, KaolinA immediately forms a sharp front with a uniformly low concentration profile beneath it. This uniform $\phi$ suspension gradually compacts through time. Particles (at $\phi=\phi_{0}$ ) rapidly aggregate to form one large attractive cluster or gel which exhibits solid-like properties at concentrations much below the jamming limit.

The evolution of the interface of the sedimenting Kaolin-A suspensions (3-B) shows two distinct regimes: a delayed collapse or consolidation regime associated with an upward drainage of water from the gelled deposit (i.e., transient condition), followed by a creeping regime associated with gradual and steady densification of a stable deposit. The delay time $\tau_{d}$ is estimated from the crossover between the horizontal and the initial slope of the consolidation phase. Prior to collapse, the gravitational forces resulted from the gel weight are entirely transferred to the interstitial fluid (here water) with negligible compressibility (with bulk modulus, $K_{w}=2.29 \mathrm{GPa}$ ). This process results in an immediate increase of the pore fluid pressure. A close view of the gel (Fig. 3-C) shows formation of evolving channels that provide a pathway for dissipation of the pore pressure through upward flow of water. The channels emerge in the lower regions of the bulk and reach the surface as the gel consolidates over time. As this upward flow erodes the channels and transports fine clay particles, volcano-like patterns form at the gel interface. The flow channels then vanish when the gel further consolidates, as sedimentation enters the creeping regime. We note that the delay time is slightly increased due to $\mathrm{NaCl}$ salt for the same initial volume fraction (Fig. 3-B). The addition of salt increases the interparticle attractive forces (e.g., Ali \& Bandyopadhyay, 2016), and thus the strength of the aggregates against the disrupting flow induced by the excess pore pressure (which is presumably the same in both experiments). Reducing particle size may also have an equivalent effect as increasing interparticle attractive forces (Seiphoori et al., 2020).

\subsubsection{General formulation}

The behavior of Kaolin-A suspensions cannot be described by the classic advectiondiffusion Eq. 2. The sharp, but low concentration, upper front exhibits virtually no dispersion. As discussed, prior to collapse the gel weight is supported by water. In the initial phase of the collapse, the gel exhibits an elastic deformation due to its own weight. As the gel structure deforms, water must be drained through the pore channels to allow further compression of the matrix. The weight of the gel exerts water pressure leading it to flow at a velocity $v_{w}$ within the gel, causing a local displacement of the solid particles' location, $w(z, t)$, with a particle velocity defined here as $v_{s}=\frac{\partial w}{\partial t}$. Using Darcy's 

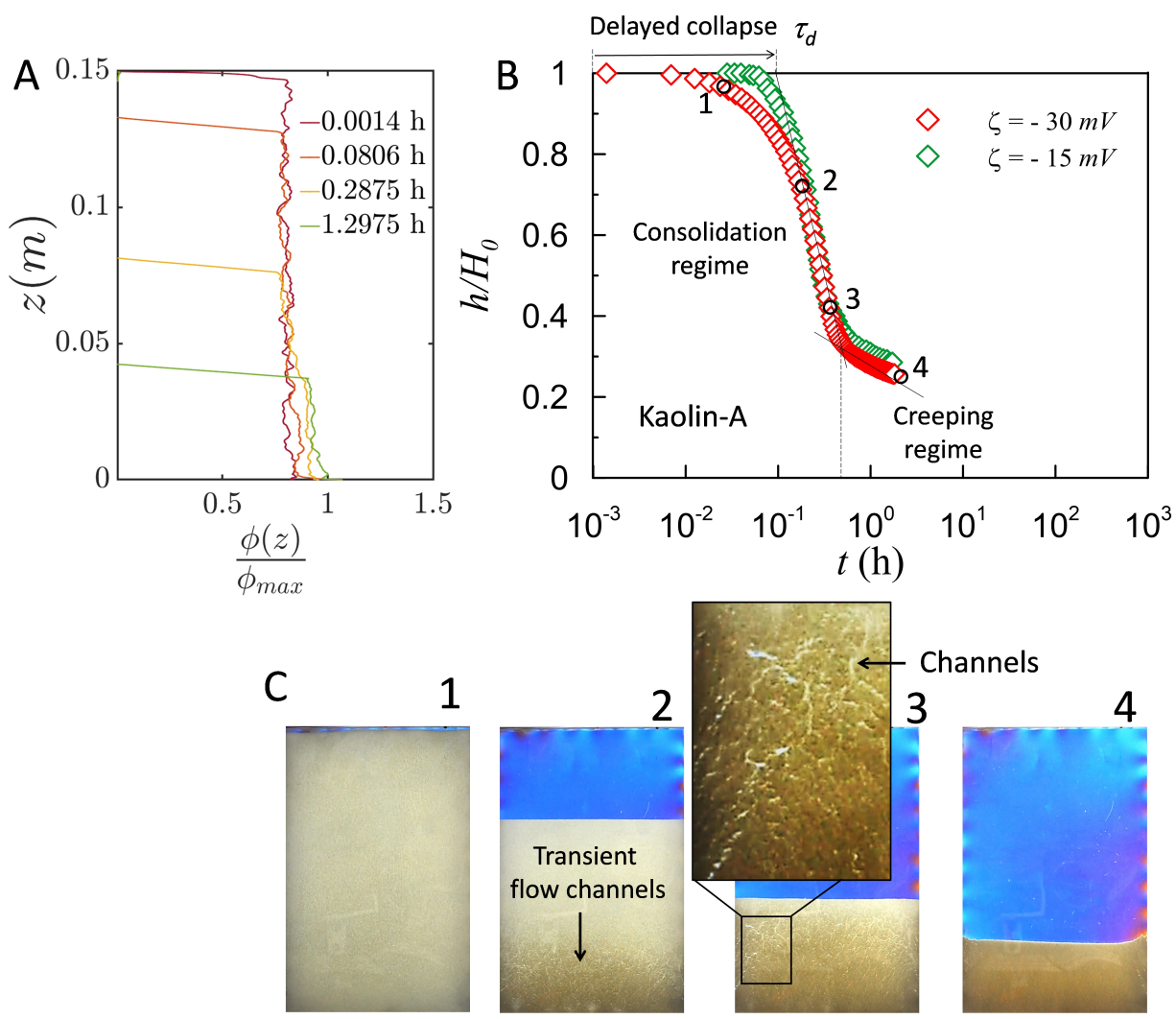

3

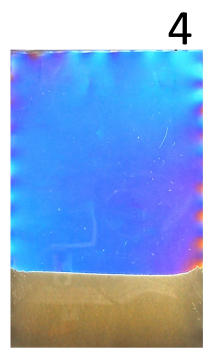

Figure 3. (A) Sedimentation profile $\frac{\phi(z)}{\phi_{\max }}$, shown at intervals of time marked in legend along the sedimentation height $z$ for attractive kaolinite (Kaolin-A) suspension $\left(\phi_{0}=1.6 \%\right)$. (B) Normalized height of the sedimenting gel interface against time for Kaolin-A suspensions with same $\phi_{0}=1.6 \%$ and different $\zeta$ potentials, demonstrating the effect of surface charge on the collapse transition time $\tau_{d}$. (C) Emerging flow channels due to upward flow of water from the bulk of the kaolinite gel, indicating the onset of consolidation and dissipation of the excess pore water. The channels vanish as sedimentation enters the creeping regime.

law for the water flow within the porous gel we have:

$$
(1-\phi)\left(v_{w}-v_{s}\right)=-\frac{k}{\mu} \frac{\partial p}{\partial z}
$$

where $p$ is the interstitial or pore water pressure, $k$ is the permeability of the gel, and $\mu$ is the water viscosity. The continuity equation for the water and solid phase results in:

$$
(1-\phi) \frac{\partial v_{w}}{\partial z}+\phi \frac{\partial v_{s}}{\partial z}=0
$$

Partial derivation of Equation 3 with respect to $z$, combining that with Equation 4 and further rearrangement results in:

$$
\frac{\partial^{2} p}{\partial z^{2}}-\frac{\mu}{k} \frac{\partial v_{s}}{\partial z}+\frac{1}{k} \frac{\partial k}{\partial z} \frac{\partial p}{\partial z}=0
$$

On the other hand, for a poroelastic gel with elastic modulus $E$, the following constitutive equation can be established:

$$
\frac{\partial v_{s}}{\partial z}=-\frac{\partial \epsilon_{z}}{\partial t}=-\frac{1}{E} \frac{\partial \sigma^{\prime}}{\partial t}
$$


where $\epsilon_{z}$ is the solid strain in $z$ direction, and $\sigma^{\prime}$ is the so called effective stress or the stress transferred by solid particles. For a fully saturated medium (such as the clay gel here), the effective stress is defined as $\sigma^{\prime} \approx \sigma_{t}-p$, where $\sigma_{t}$ is the total stress including the total weight of the gel and any externally applied load on its structure, if present. Combining Equations 5 and 6, we have:

$$
\frac{\partial^{2} p}{\partial z^{2}}+\frac{\mu}{k E}\left(\frac{\partial \sigma_{t}}{\partial t}-\frac{\partial p}{\partial t}\right)+\frac{1}{k} \frac{\partial k}{\partial z} \frac{\partial p}{\partial z}=0
$$

which leads us to a general equation describing the sedimentation and consolidation behavior of a saturated gel under a total stress of $\sigma_{t}$.

\subsubsection{Link to classic consolidation theory}

For a consolidating gel with a constant external stress over time (i.e., $\frac{\partial \sigma_{t}}{\partial t}=0$ ) and assuming that $E$ and $k$ are constant within the range of applied stresses, Equation 7 can be simplified to a diffusion equation:

$$
\frac{\partial p}{\partial t}-c_{v} \frac{\partial^{2} p}{\partial z^{2}}=0
$$

where $c_{v}=\frac{k E}{\mu}$ is termed as the coefficient of consolidation indicating the rate at which a saturated clay undergoes one-dimensional consolidation when subject to an increase in mechanical stress $\sigma$. For non-swelling clays such as kaolinite and illite, $c_{v}$ increases with an increase in mechanical stress (Robinson \& Allam, 1998). This equation, which signifies diffusion of the excess pore pressure in a soil layer, is referred to as the "1-D consolidation equation" in soil mechanics (Terzaghi, 1925; Biot, 1941). Natural depositions in rivers or seas usually involve self-weight sedimentation known as "intrinsic consolidation" (Burland, 1990). During sedimentation, initially weak clay gels consolidate to evolve into a stronger, more stable gel that creeps over time. In response to an externally applied mechanical stress, however, excess pore fluid pressure builds up - again leading the gel to undergo further consolidation to achieve stability. Such excess pore pressure can be also generated by increasing temperature or ionic strength, known as "chemical" or "thermal" consolidation (e.g., Kaczmarek \& Hueckel, 1998).

\subsubsection{Modeling kaolinite gel behavior}

In our experiments, $\phi<<1-\phi$, and thus Equation 4 implies that $v_{w} \approx 0$. At $\mathrm{t}=0$, the clay gel deformation and thus the elastic stress $\sigma$ is negligible so that $\partial p / \partial z=$ $-\Delta \rho g \phi$. According to Equation 3, permeability of the gel at the initial stage of the collapse can be formulated as $k_{0}=-\frac{\mu}{\Delta \rho g \phi} v_{s}$. We measure the initial velocity of the collapsing interface of the kaolinite gel $(\partial w / \partial t)$ at different $\phi_{0}$ to estimate the initial permeability. Manley et al (2005) proposed a scaling relation for the permeability of colloidal gels assuming that the characteristic pore size is controlled by the cluster size, thus $k_{0} \sim \frac{a^{2}}{\phi^{2 /\left(3-d_{f}\right)}}$, where $d_{f}$ is the fractal dimension $\left(d_{f} \approx 2\right)$ (Dinsmore et al., 2006). We plot previously published dimensionless permeability data for various colloidal gels formed by attractive spheres of radius $a$ (Fig. 4-A). In addition, we plot data reported by Pane and Schiffman (1997) on an attractive kaolinite clay (with $80 \%$ of the particles smaller than $2 \mu \mathrm{m}$ diameter) at relatively high $\mathrm{NaCl}$ concentration $(0.39 \mathrm{~mol} / \mathrm{L})$. Considering the plate-like morphology of kaolinite particles, we plot the scaled permeability for our experiments considering both the thickness $(d \sim 1 \mu \mathrm{m})$ and width $(d \sim 10 \mu \mathrm{m})$ of a typical kaolinite tactoid. Using tactoid thickness as the relevant particle diameter, we find that the scaled permeability of our kaolinite gels are consistent with all data, which follow the curve predicted by Manley et al. (2005) (Fig. 4-A). If instead we choose particle width (rather than thickness) for Kaolin-A experiments, permeability values are underestimated and are closer to the prediction of the semiempirical Carmen-Kozeny relation (Carman, 1956). 
For a short time after the test begins (i.e., $t<\tau_{d}$ ), the gel's weight is supported by the water pressure, while at long time (i.e., during the creeping regime) it is balanced by the gel's elasticity. Both play a role during the consolidation phase, as the excess pore pressure dissipates, and thus the following constitutive relationship can be established for a poroelastic gel:

$$
\sigma=E \frac{\partial w}{\partial z}-(1-\phi) p
$$

The stress gradient in the gel is balanced by gravity so that $\partial \sigma / \partial z=-\Delta \rho g \phi$. The initial and boundary conditions with respect to our sedimentation column can be written as $w(z, 0)=0, w(0, t)=\frac{\partial p}{\partial z}=0$. Furthermore, $p=E \frac{\partial w}{\partial z}=0$ at $z=h(t)$, where the hydrostatic pressure is negligible (see Figure $3-\mathrm{C}$ ). For these initial and boundary conditions, Equations 3, 4, and 9 can be solved for $h(z, t)$ using separation of variables:

$$
h_{0}-h(t)=\Delta h\left(1-e^{-t / \tau}\right)
$$

where the total change of the height is $\Delta h=\frac{\Delta \rho g \phi h_{0}^{2}}{2 E}$, and the time scale for the consolidation is $\tau=\frac{4 \mu(1-\phi) h_{0}^{2}}{\pi^{2} k_{0} E}$ (Manley et al., 2005). Experimental results and the model performance are presented in Figure 4-B and $\mathrm{C}$ in terms of the variation of the sedimenting interface versus time at various initial volume fractions. We find that the dynamics of all gels are well described by the model, despite the range of consolidation timescales spanning two orders of magnitude. This is illustrated by the data collapse in Figure 4$\mathrm{C}$ using the non-dimensional form of Equation 10, where $\hat{h}=\left(h_{0}-h\right) / \Delta h$ and $\hat{t}=$ $t / \tau$. By introducing a weak concentration of $\mathrm{NaCl}$ in solution at low- $\phi$, we find that the delay time for gel collapse increases by an order of magnitude but the gel elasticity and permeability (and therefore dynamics) barely change.

\section{Conclusions}

It is well known that sedimentation of natural mud and kaolinite suspensions deviates from classic hard-sphere behavior due to aggregation. Our experiments are consistent with previous observations in this regard. We go one step further, however, by showing how manipulation of surface charge may drive kaolinite clay from an attractive to repulsive suspension, recovering many aspects of classical sedimentation behavior. Although the transition is likely gradual, it appears that a zeta potential value of $\zeta \approx-30$ $\mathrm{mV}$ separates attractive from repulsive behavior for kaolinite in water in our experiments. Kaolinite suspended in de-ionized water exhibits significant aggregation, which is gradually enhanced through the addition of $\mathrm{NaCl}$ and the associated increase in zeta potential. The addition of $\left(\mathrm{NaPO}_{3}\right)_{6}$, sharply lowers zeta potential and suppresses aggregation entirely. Repulsive suspensions of silica spheres and kaolinite exhibit qualitatively similar dynamics; in particular, the formation of a diffuse sigmoidal concentration profile, and sedimentation dynamics that are broadly consistent with classical hindered settling. Kaolinite sedimentation rates, however, are two orders of magnitude slower than silica spheres having similar diameter and zeta potential. The difference in average sedimentation rates of the respective fronts can be explained by the influence of mass and shape on the fall velocity of individual particles. The substantially larger dispersion coefficient for kaolinite (three orders of magnitude), however, hints at more complex shape effects. Recent research has demonstrated that lubrication effects are of paramount importance in determining sedimentation rates of dense suspensions (Brzinski \& Durian, 2018), and it is expected that changes in shape will strongly influence lubrication forces (Chong et al., 1979). We postulate that the plate-like shape of kaolinite particles is enhancing lubrication and dispersion, perhaps somehow associated with alignment of particles through hydrodynamic interactions (Chong et al., 1979; Le Roux, 2004). Sedimentation kinematics indicate that kaolinite plates are aligned with their long axis orthogonal to the settling (vertical) direction. Future work that includes direct microscopic observation would provide valuable insight on the coupled roles of shape and lubrication. 
A

$\begin{array}{ll}\text { - } & \text { Silica, } a=10 \mathrm{~nm} \\ & \text { Calcium Carbonate, } a=35 \mathrm{~nm} \\ \text { - Silica, } a=50 \mathrm{~nm} \\ -\quad \text { PMMA, } a=186 \mathrm{~nm} \\ \diamond \quad \text { Kaolin-A }(a=d / 2 \sim 0.5 \mu \mathrm{m}) \\ \diamond \quad \text { Kaolin-A }(a=D / 2 \sim 5 \mu \mathrm{m}) \\ \diamond \quad \text { Kaolinite }(a=d / 2 \sim 0.5 \mu, \text { Pane and Schiffman, 1997) } \\ --- & \text { Carman-Kozeny }\end{array}$

Fractal cluster size (Manley et al., 2005)

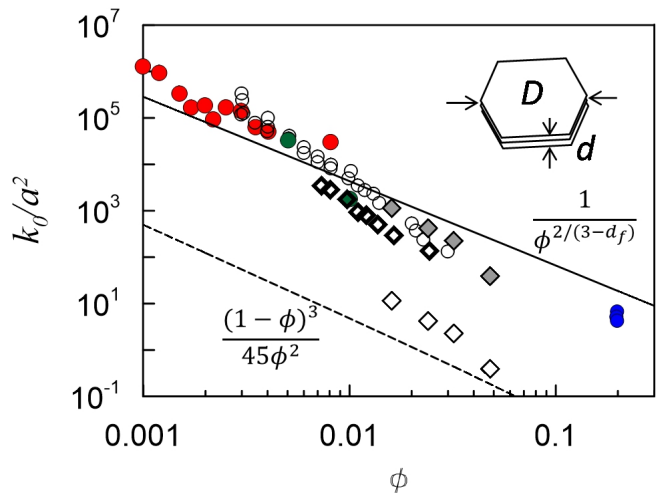

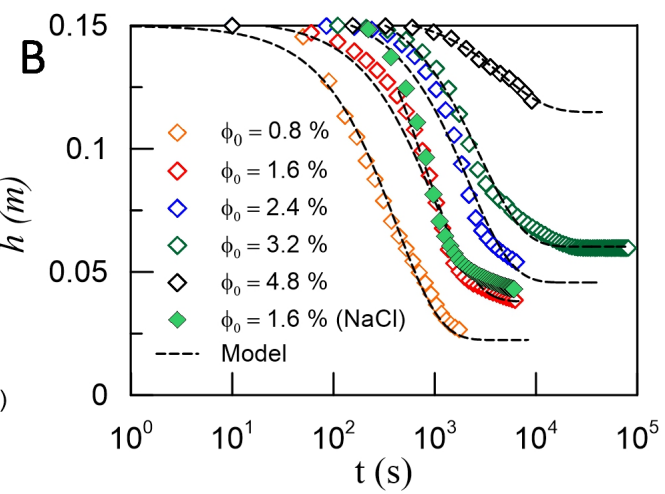

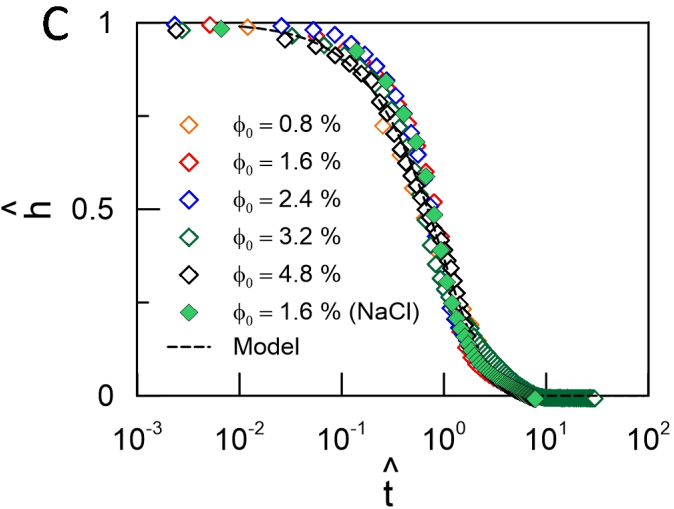

Figure 4. (A) Scaled permeability of the gel's elastic network estimated from the velocity of the collapsing interface at initial stage of compression for various colloidal gels including: silica spheres of diameters 20 and $50 \mathrm{~nm}$ (Manley et al., 2005), calcium carbonate of diameter $70 \mathrm{~nm}$ (Allain et al., 1995), PMMA spheres of diameter $0.37 \mu \mathrm{m}$ (Starrs et al., 2002b), and kaolinite particles assuming the thickness $(d \sim 1 \mu \mathrm{m})$ of a typical kaolinite tactoid. (B) Experimental results showing the variation of the gel's interface over time for various initial volume fractions and the proposed model. (C) Experimental results collapsed on a master model.

Attractive kaolinite suspensions appear to immediately form large aggregated clusters even at fairly low concentrations $\left(\phi_{0}<2 \%\right)$, which display characteristics of a soft solid. Kaolinite suspensions exhibit similar dynamics to colloidal gels formed by distinctly different surface interactions (Starrs et al., 2002a), suggesting that recent progress in the formation and rheology of idealized gels may be transferable to natural mud suspensions (Jerolmack \& Daniels, 2019), including other clays such as montmorillonite (Marr et al., 2001). The evolution of the sedimenting interface in clay gel indicates two distinct regimes of transitional collapse or consolidation, associated with dissipation of the excess interstitial fluid pressure followed by a creep regime associated with mechanical densification of the deposit.

The consolidation regime is characterized by formation of flow channels in the bulk of the gel. These channel patterns are an interesting mesoscale feature of the transient collapse process; they indicate a kind of fracturing of the bulk that focuses drainage, yet they do not disrupt the applicability of a continuum macroscopic framework (as driven in this paper). These drainage features appear similar to pipes and fluid-escape structures that commonly form in soft sediment deposits (Nocita, 1988; Owen et al., 2011; Brogi et al., 2017; Wheatley \& Chan, 2018). These patterns typically have the form of sheet dewatering structures or fluid conduits and veins (Brogi et al., 2017; Marr et al., 
2001; Hrabovszki et al., 2020). Features such as calcite veins associated with hydrothermal eruption, and similar fluid-escape processes, are used to estimate hydrothermal origins of the parent fluid. Understanding the conditions that lead to their formation may aid in interpreting the significance of these features in the rock record.

A major challenge is that sedimentation dynamics appear to be exquisitely sensitive to surface charge and particle shape effects. Linking the mechanics of grain-scale particle-particle and particle-fluid interactions — such as friction, lubrication and cohesion - to bulk rheology is essential to make progress toward more predictive (rather than empirical) models for sedimentation and flow of mud suspensions (Bonn et al., 2017; Basu et al., 2014). These findings have consequences for the sedimentation and erosion of deposits in natural environments, as the presence of salt is ubiquitous. The increased $\mathrm{NaCl}$ concentration of seawater relative to freshwater is a major contributor to aggregation and sedimentation of mud in estuaries and coastal environments (Winterwerp, 2002). Some artificial fertilizers, however, contain salts that are functionally similar to sodium hexametaphosphate (Litke, 1999), which would act to suppress aggregation. Interaction of clay particles and organic materials in natural environments can create further complex scenarios such as the flocculation by polymeric organic molecules that promote clay aggregation and deposition rates (Zeichner et al., 2021). The kaolinite gels formed in the laboratory under pure sedimentation known as "intrinsic consolidation" are fragile solids that eventually consolidate. We hypothesize that gentle hydrodynamic shearing may be sufficient to prevent this collapse, and maintain a meta-stable gel that corresponds to fluid muds observed in many natural settings (Parsons et al., 2001). Experiments that introduce such a shear (Nie et al., 2020), while obtaining spatially-resolved maps of concentration, strain and structure within the gel, are a next logical step.

\section{Acknowledgments}

The authors would like to thank K.L. Galloway for fruitful discussions and ARO W911NF-16-1-0290 for financial support.

Data Availability Statements. Experimental data and the supplementary movies (S1S14) for this study are publicly available at https://zenodo.org/record/4586801.

\section{References}

Ali, S., \& Bandyopadhyay, R. (2016). Aggregation and stability of anisotropic charged clay colloids in aqueous medium in the presence of salt. Faraday discussions, 186, 455-471.

Allain, C., Cloitre, M., \& Wafra, M. (1995). Aggregation and sedimentation in colloidal suspensions. Physical review letters, 74 (8), 1478.

Bartlett, P., Teece, L. J., \& Faers, M. A. (2012). Sudden collapse of a colloidal gel. Physical Review E, 85(2), 021404.

Basu, A., Xu, Y., Still, T., Arratia, P., Zhang, Z., Nordstrom, K., ... Yodh, A. (2014). Rheology of soft colloids across the onset of rigidity: scaling behavior, thermal, and non-thermal responses. Soft matter, 10(17), 3027-3035.

Bergström, L. (1992). Sedimentation of flocculated alumina suspensions: $\gamma$-ray measurements and comparison with model predictions. Journal of the Chemical Society, Faraday Transactions, 88(21), 3201-3211.

Biot, M. A. (1941). Consolidation settlement under a rectangular load distribution. Journal of Applied Physics, 12(5), 426-430.

Bonn, D., Denn, M. M., Berthier, L., Divoux, T., \& Manneville, S. (2017). Yield stress materials in soft condensed matter. Reviews of Modern Physics, 89(3), 035005.

Brogi, A., Capezzuoli, E., Kele, S., Baykara, M. O., \& Shen, C.-C. (2017). Key travertine tectofacies for neotectonics and palaeoseismicity reconstruction: ef- 
fects of hydrothermal overpressured fluid injection.

Journal of the Geological Society, 174(4), 679-699.

Brzinski, T. A., \& Durian, D. J. $\quad$ (2018, Dec). Observation of two branches in the hindered settling function at low reynolds number. Phys. Rev. Fluids, 3, 124303.

Burland, J. (1990). On the compressibility and shear strength of natural clays. Géotechnique, 40(3), 329-378.

Buscall, R., Choudhury, T. H., Faers, M. A., Goodwin, J. W., Luckham, P. A., \& Partridge, S. J. (2009). Towards rationalising collapse times for the delayed sedimentation of weakly-aggregated colloidal gels. $\quad$ Soft Matter, 5(7), 13451349.

Carman, P. C. (1956). Flow of gases through porous media.

Chong, Y. S., Ratkowsky, D. A., \& Epstein, N. (1979). Effect of particle shape on hindered settling in creeping flow. Powder Technology, 23(1), 55-66.

Coussot, P. (2017). Mudflow rheology and dynamics. Routledge.

Coussot, P., \& Piau, J. M. (1994). On the behavior of fine mud suspensions. Rheologica acta, 33(3), 175-184.

Dankers, P., \& Winterwerp, J. (2007). Hindered settling of mud flocs: theory and validation. Continental shelf research, 27(14), 1893-1907.

Derec, C., Senis, D., Talini, L., \& Allain, C. (2003). Rapid settling of a colloidal gel. Physical Review E, 67(6), 062401.

Dinsmore, A., Prasad, V., Wong, I., \& Weitz, D. (2006). Microscopic structure and elasticity of weakly aggregated colloidal gels. Physical review letters, 96(18), 185502.

Edzwald, J. K., \& OMelia, C. R. (1975). Clay distributions in recent estuarine sediments. Clays and Clay minerals, 23(1), 39-44.

Goldberg, S., \& Sposito, G. (1985). On the mechanism of specific phosphate adsorption by hydroxylated mineral surfaces: A review. Communications in Soil Science and Plant Analysis, 16(8), 801-821.

Gopalakrishnan, V., Schweizer, K. S., \& Zukoski, C. (2006). Linking single particle rearrangements to delayed collapse times in transient depletion gels. Journal of Physics: Condensed Matter, 18(50), 11531.

Guazzelli, É., Morris, J., \& Pic, S. (2011). A physical introduction to suspension dynamics. Cambridge University Press.

Heymann, L., Peukert, S., \& Aksel, N. (2002). On the solid-liquid transition of concentrated suspensions in transient shear flow. Rheologica acta, 41 (4), 307-315.

Hrabovszki, E., Tóth, E., Tóth, T. M., Máthé, Z., \& Schubert, F. (2020). Potential formation mechanisms of early diagenetic displacive veins in the permian boda claystone formation. Journal of Structural Geology, 138, 104098.

Jerolmack, D. J., \& Daniels, K. E. (2019). Viewing earths surface as a soft-matter landscape. Nature Reviews Physics, 1-15.

Johnson, H. B., \& Kessler, F. (1969). Kaolinite dehydroxylation kinetics. Journal of the American Ceramic Society, 52(4), 199-203.

Kaczmarek, M., \& Hueckel, T. (1998). Chemo-mechanical consolidation of clays: analytical solutions for a linearized one-dimensional problem. Transport in porous media, 32(1), 49-74.

Kretzschmar, R., Sticher, H., \& Hesterberg, D. (1997). Effects of adsorbed humic acid on surface charge and flocculation of kaolinite. Soil Science Society of America Journal, 61(1), 101-108.

Kynch, G. J. (1952). A theory of sedimentation. Transactions of the Faraday society, 48, 166-176.

Le Roux, J. (2004). A hydrodynamic classification of grain shapes. Journal of Sedimentary Research, $74(1), 135-143$.

Litke, D. W. (1999). Review of phosphorus control measures in the united states and their effects on water quality. Water-Resources Investigations Report, 99, 
4007.

Manley, S., Skotheim, J., Mahadevan, L., \& Weitz, D. A. (2005). Gravitational collapse of colloidal gels. Physical review letters, 94(21), 218302.

Marr, J. G., Harff, P. A., Shanmugam, G., \& Parker, G. (2001). Experiments on subaqueous sandy gravity flows: The role of clay and water content in flow dynamics and depositional structures. Geological Society of America Bulletin, $113(11), 1377-1386$.

Martin, J., Rakotomalala, N., \& Salin, D. (1994). Hydrodynamic dispersion broadening of a sedimentation front. Physics of Fluids, 6(10), 3215-3217.

McAnally, W. H., Friedrichs, C., Hamilton, D., Hayter, E., Shrestha, P., Rodriguez, H., ... Teeter, A. (2007). Management of fluid mud in estuaries, bays, and lakes. i: Present state of understanding on character and behavior. Journal of Hydraulic Engineering, 133(1), 9-22.

Mitchell, J. K., \& Soga, K. (2005). Fundamentals of soil behavior (Vol. 3). John Wiley \& Sons New York.

Mueller, S., Llewellin, E., \& Mader, H. (2010). The rheology of suspensions of solid particles. Proceedings of the Royal Society A: Mathematical, Physical and Engineering Sciences, 466(2116), 1201-1228.

Nie, S., Jiang, Q., Cui, L., \& Zhang, C. (2020). Investigation on solid-liquid transition of soft mud under steady and oscillatory shear loads. Sedimentary Geology, 397, 105570.

Nocita, B. W. (1988). Soft-sediment deformation (fluid escape) features in a coarsegrained pyroclastic-surge deposit, north-central new mexico. Sedimentology, $35(2), 275-285$.

Owen, G., Moretti, M., \& Alfaro, P. (2011). Recognising triggers for soft-sediment deformation: current understanding and future directions. Sedimentary Geology, 235(3-4), 133-140.

Packman, A., \& Jerolmack, D. (2004). The role of physicochemical processes in controlling sediment transport and deposition in turbidity currents. Marine Geology, $204(1-2), 1-9$.

Parsons, J. D., Whipple, K. X., \& Simoni, A. (2001). Experimental study of the grain-flow, fluid-mud transition in debris flows. The Journal of Geology, $109(4), 427-447$.

Pauchard, L., Parisse, F., \& Allain, C. (1999). Influence of salt content on crack patterns formed through colloidal suspension desiccation. Physical Review E, $59(3), 3737$.

Robinson, R. G., \& Allam, M. M. (1998). Effect of clay mineralogy on coefficient of consolidation. Clays and clay minerals, 46(5), 596-600.

Seiphoori, A., Ma, X.-g., Arratia, P. E., \& Jerolmack, D. J. (2020). Formation of stable aggregates by fluid-assembled solid bridges. Proceedings of the National Academy of Sciences, 117(7), 3375-3381.

Starrs, L., Poon, W., Hibberd, D., \& Robins, M. (2002b). Collapse of transient gels in colloid-polymer mixtures. Journal of Physics: Condensed Matter, 14(10), 2485 .

Starrs, L., Poon, W. C. K., Hibberd, D. J., \& Robins, M. M. (2002a). Collapse of transient gels in colloid-polymer mixtures. Journal of Physics: Condensed Matter, $14(10), 2485$.

Sutherland, B. R., Barrett, K. J., \& Gingras, M. K. (2015). Clay settling in fresh and salt water. Environmental Fluid Mechanics, 15(1), 147-160.

Talling, P. J., Masson, D. G., Sumner, E. J., \& Malgesini, G. (2012). Subaqueous sediment density flows: Depositional processes and deposit types. Sedimentology, 59(7), 1937-2003.

Teece, L. J., Hart, J. M., Hsu, K. Y. N., Gilligan, S., Faers, M. A., \& Bartlett, P. (2014). Gels under stress: The origins of delayed collapse. Colloids and Surfaces A: Physicochemical and Engineering Aspects, 458, 126-133. 
Terzaghi, K. (1925). Erdbaumechanik auf bodenphysikalischer grundlage.

Toorman, E. A., \& Berlamont, J. E. (1991). A hindered settling model for the prediction of settling and consolidation of cohesive sediment. Geo-marine letters, 11(3-4), 179-183.

Traykovski, P., Geyer, W. R., Irish, J., \& Lynch, J. (2000). The role of wave-induced density-driven fluid mud flows for cross-shelf transport on the eel river continental shelf. Continental Shelf Research, 20(16), 2113-2140.

Wheatley, D. F., \& Chan, M. A. (2018). Clastic pipes and soft-sediment deformation of the jurassic carmel formation, southern utah, usa: implications for pipe formation mechanisms and host-rock controls. Journal of Sedimentary Research, 88(9), 1076-1095.

Whitehouse, U. G., Jeffrey, L. M., \& Debbrecht, J. D. (2013). Differential settling tendencies of clay minerals in saline waters. In (pp. 1-79). Elsevier.

Winterwerp, J. C. (2002). On the flocculation and settling velocity of estuarine mud. Continental shelf research, 22(9), 1339-1360.

Zeichner, S. S., Nghiem, J., Lamb, M. P., Takashima, N., de Leeuw, J., Ganti, V., \& Fischer, W. W. (2021). Early plant organics increased global terrestrial mud deposition through enhanced flocculation. Science, 371(6528), 526-529. 